\title{
ON COMPACT ITÔ'S FORMULAS FOR MARTINGALES OF $m_{c}^{4}$
}

\author{
MaRIa Jolis
}

Abstract

We prove that the class $m_{c}^{4}$ of continuous martingales with parameter set $[0,1]^{2}$, bounded in $L^{4}$, is included in the class of semi-martingales $S_{c}^{\infty}\left(L_{0}(P)\right)$ defined by Allain in $[A]$. As a consequence we obtain a com. pact Itô's formula. Finally we relate this result with the compact Itô formula obtained by Sanz in [S] for martingales of $\boldsymbol{m}_{\mathbf{c}}^{\mathbf{4}}$.

\section{Introduction}

The purpose of this work is to find a relationship between the compact Itô's formulas in the plane given by M. F. Allain and M. Sanz (see $[\mathbf{A}]$ and $[\mathbf{S}]$ ). Before presenting the main result we introduce the problem of Itô's formulae in the plane.

Recall that when we consider a 1-dimensional parameter, if $X_{t}=X_{0}+M_{t}+B_{t}$ is a continuous semimartingale (that is $E\left|X_{0}\right|<\infty, M_{t}$ is a continuous square integrable martingale and $B_{t}$ is a continuous process of total variation integrable on any finite interval) and $F \in \mathcal{C}^{2}(\boldsymbol{F})$ then

$$
F\left(X_{t}\right)=F\left(X_{0}\right)+\int_{0}^{t} F^{\prime}\left(X_{s}\right) d M_{s}+\int_{0}^{t} F^{\prime}\left(X_{s}\right) d B_{s}+\frac{1}{2} \int_{0}^{t} F^{\prime \prime}\left(X_{s}\right) d<M>_{s} .
$$

This expression is known as the Itô formula for $F(X)$.

The idea of the proof consists in taking a sequence of partitions of the interval $[0, t]: \mathcal{P}^{n}=\left\{0=t_{0}^{n}<\cdots<t_{p_{n}}^{n}<t\right\}$ with $\left|\mathcal{P}^{n}\right|=\sup _{i=1, \ldots, p_{n}+1}\left|t_{i}^{n}-t_{i-1}^{n}\right|$ tending to 0 when $n \rightarrow \infty\left(t_{p_{n}+1}^{n}=t\right)$, and write

$$
\begin{gathered}
F\left(X_{t}\right)-F\left(X_{0}\right)=\sum_{i=0}^{p_{n}} F\left(X_{i_{i+1}^{n}}\right)-F\left(X_{t_{i}^{n}}\right)=\sum_{i=0}^{p_{n}} F^{\prime}\left(X_{t_{i}^{n}}\right)\left(X_{i_{i+1}^{n}}-X_{i_{i}^{n}}\right)+ \\
\frac{1}{2} \sum_{i=0}^{p_{n}} F^{\prime \prime}\left(X_{i_{i}^{n}}\right)\left(X_{t_{i+1}^{n}}-X_{i_{i}^{n}}\right)^{2}+\sum_{i=0}^{p_{n}} r\left(X_{i_{i+1}^{n}}, X_{i_{i}^{n}}\right)
\end{gathered}
$$


where we have applied Taylor's formula and $r\left(X_{t_{i+1}^{n}}, X_{t_{i}^{n}}\right)$ represents the error term. Then, it can be proved that the first two terms tend to the integrals

$$
\int_{0}^{t} F^{\prime}\left(X_{s}\right) d M_{s}+\int_{0}^{t} F^{\prime}\left(X_{s}\right) d B_{s} \quad \text { and } \quad \int_{0}^{t} F^{\prime \prime}\left(X_{s}\right) d<M>_{s}
$$

respectively and the error term tends to 0 , when $\left|\mathcal{P}^{n}\right| \downarrow 0$.

In the two-parameter case the idea would be to take a sequence of partitions of the rectangle $R_{s t}, \mathcal{P}^{n}=\mathcal{P}_{1}^{n} \times \mathcal{P}_{2}^{n}$ where

$$
\begin{array}{cc}
\mathcal{P}_{1}^{n}=\left\{0=s_{1}^{n}<\cdots<s_{p_{n}}^{n}<s\right\} & , \quad s_{p_{n}+1}^{n}=s, \\
\mathcal{P}_{2}^{n}=\left\{0=t_{1}^{n}<\cdots<t_{q_{n}}^{n}<t\right\} \quad, \quad s_{q_{n}+1}^{n}=t .
\end{array}
$$

By convenience we remove the index $n$, putting $u$ for a generic point $\left(s_{i}, t_{j}\right)$ of the partition $\mathcal{P}$ and $\Delta_{u}$ for the rectangle $\left(s_{i}, s_{i+1}\right] \times\left(t_{j}, t_{j+1}\right]$ and $\bar{u}$ for $\left(s_{i+1}, t_{j+1}\right)$. Assuming that the process vanishes on the axes and that $F(0)=0$, we write

$$
F\left(X_{s t}\right)=\sum_{u \in \mathcal{P}}(F \circ X)\left(\Delta_{u}\right)
$$

where the increment of a function in a rectangle is defined by

$$
f\left(\Delta_{u}\right)=f\left(s_{i}, t_{j}\right)+f\left(s_{i+1}, t_{j+1}\right)-f\left(s_{i}, t_{j+1}\right)-f\left(s_{i+1}, t_{j}\right),
$$

and then we take in the right hand side the limit when $|P| \rightarrow 0$.

Here two problems appear. The first one is to determine the class of processes for which a Itô's formula will be valid. In the one-parameter case, from Itô's formula, we have that the class of continuous semimartingales is closed for the composition with functions $F \in \mathcal{C}^{2}$. In the two-parameter case, it is not clear what definition of semimartingale should be taken ( see for instance [A], and also [12]). We will follow the approach of M. F. Allain ([A]). Most of the results obtained up to date, are Itô's formulae for martingales, but of course we can not expect $F(M)$ to be a martingale.

The second problem that appears is how to apply Taylor's formula to (1) and how to compute and identify the limits that we should obtain. This question has led different authors to consider the problem of obtaining an Itô's formula in a different way, that is, first fixing a parameter and aplying the ordinary Ito's formula for the martingale that is obtained varying the other parameter. Then they consider the integrals that appear like limits when the norm of the partition tends to 0 of some Riemman's sums. These Riemann sums are developed by using again the one parameter Itô formula. These formulae are called "developed" Itô's formulae and have been proved, among others, by Chevalier (see [Ch]) and Nualart (see [N2]). The last one is the most general and the result obtained is as follows. 
Itô's formula. (Nualart)(see [N2]). Let $f: \mathbb{R} \rightarrow \mathbb{R}$ be a function of class $\mathcal{C}^{4}$ that vanishes at 0 and let $M$ be a martingale in the space $m_{c}^{4}$ (continuous martingales bounded in $L^{4}$ null on the axes). Then for any $(s, t) \in \mathbf{R}_{+}^{2}$ we have

$$
\begin{aligned}
f\left(M_{s t}\right)= & \int_{R_{s t}} f^{\prime}\left(M_{z}\right) d M_{z}+\int_{R_{s t}} f^{\prime \prime}\left(M_{z}\right) d \tilde{M}_{z}+ \\
& \frac{1}{2} \int_{0}^{s} f^{\prime \prime}\left(M_{x t}\right) d<M_{. t}>_{x}+\frac{1}{2} \int_{0}^{t} f^{\prime \prime}\left(M_{s y}\right) d<M_{s .}>_{y}- \\
& \left.\frac{1}{2} \int_{R_{s t}} f^{\prime \prime}\left(M_{z}\right) d<M_{z}-\int_{R_{s t}} f^{\prime \prime \prime}\left(M_{z}\right) d<M, \tilde{M}\right\rangle_{z}- \\
& \left.\frac{1}{4} \int_{R_{s t}} f^{I V}\left(M_{z}\right) d<\tilde{M}\right\rangle_{z} .
\end{aligned}
$$

The process $\tilde{M}_{z}$ is defined by $\dot{M}_{z}=\lim _{n} \sum_{u \in \mathcal{P}_{z}} M\left(\Delta_{u}^{1}\right) M\left(\Delta_{u}^{2}\right)$, where $\mathcal{P}_{z}$ is the minimal partition that contains $\mathcal{P}$ and $z=(s, t)$ (in [N1] the existence of $\tilde{M}_{z}$ is proved as a uniform limit in $L^{2}, \tilde{M}_{z}$ being a continuous martingale) and $\Delta_{u}^{1}$ and $\Delta_{u}^{2}$ are the rectangles given by

$$
\Delta_{u}^{1}=\left(s_{i}, s_{i+1}\right] \times\left(0, t_{j}\right], \quad \Delta_{u}^{2}=\left(0, s_{i}\right] \times\left(t_{j}, t_{j+1}\right]
$$

On the other hand $\langle M\rangle_{z}=\lim \sum_{u \in \mathcal{P}_{z}} M\left(\triangle_{u}\right)^{2}$ (in [N1] it is also showed that if $M \in m_{c}^{2}$ this limit is in $L^{1}(\Omega)$ and that the process $\langle M\rangle_{z}$ has continuous paths).

The formulas that are obtained directly by applying (in a suitable way) Taylor's formula in (1) are called "compact" Itô's formulae. The idea is to apply Taylor's formula in the following way (see $[\mathbf{A}]$ where this problem is considered with m-dimensional parameter and also [I1]).

If $u=\left(u_{1}, u_{2}\right)$ and $v=\left(v_{1}, v_{2}\right)$ we denote by $u \otimes v$ the point $\left(u_{1}, v_{2}\right)$. Then

$$
f(M)\left(\Delta_{u}\right)=\left[f\left(M_{\bar{u}}\right)-f\left(M_{u}\right)\right]-\left[f\left(M_{u \otimes \bar{u}}\right)-f\left(M_{u}\right)\right]-\left[f\left(M_{\bar{u} \otimes u}\right)-f\left(M_{u}\right)\right]
$$

and so we can write

$$
\begin{aligned}
& f\left(M_{z}\right)=\sum_{u \in \mathcal{P}_{\bar{z}}} f(M)\left(\Delta_{u}\right)= \\
& \left.\sum_{u \in \mathcal{P}_{\bar{z}}}\left\{f\left(M_{\bar{u}}\right)-f\left(M_{u}\right)\right]-\left[f\left(M_{u \otimes \bar{u}}\right)-f\left(M_{u}\right)\right]-\left[f\left(M_{\bar{u} \otimes u}\right)-f\left(M_{u}\right)\right]\right\}= \\
& \sum_{1 \leq r \leq 4} \frac{1}{r !} \sum_{u \in \mathcal{P}_{\bar{z}}} f^{(r)}\left(M_{u}\right)\left\{\left(M_{\bar{u}}-M_{u}\right)^{r}-\left(M_{u \otimes u ̈}-M_{u}\right)^{r}-\left(M_{\bar{u} \otimes u}-M_{u}\right)^{r}\right\}+ \\
& \sum_{u \in \mathcal{P}_{z}} R(f, u)
\end{aligned}
$$


where $R(f, u)$ are the error terms of Taylor's formula and we have to consider $|\mathcal{P}| \downarrow 0$.

Set $\Delta_{u}^{r}(M)=\left(M_{\bar{u}}-M_{u}\right)^{r}-\left(M_{u \otimes \bar{u}}-M_{u}\right)^{r}-\left(M_{\bar{u} \otimes u}-M_{u}\right)^{r}=$ $\left[M\left(\Delta_{u}\right)+M\left(\Delta_{u}^{1}\right)+M\left(\Delta_{u}^{2}\right)\right]^{r}-M\left(\Delta_{u}^{1}\right)^{r}-M\left(\Delta_{u}^{2}\right)^{r}$.

The processes obtained as $\bar{M}_{z}^{(r)}=P-\lim _{n} \sum_{u \in \mathcal{P}_{z}} \Delta_{u}^{r}(M)$ are called $r$ variations of the process $M$.

M. Sanz shows in [S] the existence and continuity, for martingales of $m_{c}^{4}$, of the $r$-variations, and also shows that for $r \geq 5$ they are equal to 0 . Furthermore for $X$ continuous and adapted she shows the existence and continuity of the processes defined by

$$
\int_{R_{x}} X_{u} d \bar{M}_{u}^{(r)}=P-\lim _{|\mathcal{P}|_{10}} \sum_{u \in \mathcal{P}_{\boldsymbol{x}}} X_{u} \Delta_{u}^{r}(M)
$$

Finally she proves the following formula.

Itô's formula 1. (Sanz) If $M \in m_{c}^{4}, f \in \mathcal{C}^{4}(\mathbb{B}), f(0)=0$ then

$$
f\left(M_{z}\right)=\sum_{r=1}^{4} \frac{1}{r !} \int_{R_{x}} f^{(r)}\left(M_{u}\right) d \bar{M}_{u}^{(r)}
$$

In order to see that the remainder terms in (2) tend to 0, Sanz shows that the terms of the right hand side of this last expression coincide with the terms of the Nualart's formula. This can be proved by using an algebraic identity and the $L_{p}$-integrator property of the 2-variation proved in [I2].

On the other hand M. F. Allain considers (2) when $|\mathcal{P}| \downarrow 0$ as the integral of the processes $f^{(r)}(M)$ with respect to some stochastic measures (we will define precisely in the following section the term stochastic measure and the notions that are used in the Ito formula of Allain). She proves the following formula.

Itô's formula 2. (Allain) If $M \in S_{c}^{\infty}\left(L_{0}(P)\right)$ is such that there exists $m \in$ $\mathbf{N} \backslash\{0\}$ such that $\forall k \geq m+1 \mu^{(k)} \equiv 0$. Then

$$
F(M)\left(\left(z, z^{\prime}\right]\right)=\sum_{i=1}^{m} \frac{1}{r !} \int_{\left(z, z^{\prime}\right]} f^{(r)}\left(M_{u}\right) d \mu^{(r)}
$$

Here $\mu^{(k)}$ are some stochastic measures obtained from the processes $M^{r}$ with $1 \leq r \leq k$ that we will define later.

Allain gives also several examples of seminartingales that belong to the class $S_{c}^{\infty}\left(L_{0}(P)\right)$ : The representable semimartingales defined by Wong and Zakai (see [WZ]), the processes with paths of class $\mathcal{C}^{2}$ and the product of two independent one-parameter martingales. 
Later, Imkeller in [I1] proved the Allain formula for $M \in m_{c, s}^{8}$ (space of continuous strong martingales bounded in $L^{g}(\Omega)$ ).

Notice that formally formulae 1 and 2 are the same. Our contribution consists in analyzing the relation between these two formulae. We carry out our study in two steps:

I. Prove that $m_{c}^{4} \subset S_{c}^{\infty}\left(L_{0}(P)\right)$. This is the main part of this work.

II. Show the total equivalence between the two Itô's formulas when $M \in$ $m_{\mathrm{c}}^{4}$.

We have structured this paper as, follows. In Section 2 we give the basic notations, the definitions and properties involved in Allain's formula. The proof of I appears in Section 3, and in Section 4 we give the proof of II. We give also an Appendix in which we quote some results on inequalities for martingales that we will use.

\section{Basic Notations and Allain's Formula}

Let $(\Omega, \mathcal{F}, P)$ be a complete probability space. The set of parameters that we consider is either $T=(0,1]^{2}$ or $\bar{T}=[0,1]^{2}$, with the partial order $(s, t) \leq\left(s^{\prime}, t^{\prime}\right)$ if and only if $s \leq s^{t}$ and $t \leq t^{t}$. By $(s, t)<\left(s^{t}, t^{t}\right)$ we mean $s<s^{\prime}$ and $t<t^{\prime}$. Given $z_{1}, z_{2} \in \bar{T}, z_{1}<z_{2},\left(z_{1}, z_{2}\right]$ denotes the rectangle $\left\{z \in \bar{T}, z_{1}<z \leq z_{2}\right\}$ (in a similar way we define $\left[z_{1}, z_{2}\right),\left[z_{1}, z_{2}\right],\left(z_{1}, z_{2}\right)$ ). Denote $(0, z]$ by $R_{z}$. If $f$ is a map from $\bar{T}$ to $\mathbb{R}$, the increment of $f$ on a rectangle $\left(z_{1}, z_{2}\right], z_{1}=\left(s_{1}, t_{1}\right), z_{2}=$ $\left(s_{2}, t_{2}\right)$ is $f\left(\left(z_{1}, z_{2}\right]\right)=f\left(z_{1}\right)-f\left(s_{1}, t_{2}\right)-f\left(s_{2}, t_{1}\right)+f\left(z_{2}\right)$.

Let $\left(\mathcal{F}_{z}\right)_{z \in \bar{T}}$ be an increasing family of sub $\sigma$-fields of $\mathcal{F}$. For any $(s, t) \in \bar{T}$ define $\mathcal{F}_{s t}^{1}=\vee v \mathcal{F}_{s v}$ and $\mathcal{F}_{s t}^{2}=\underset{v}{\vee} \mathcal{F}_{u t}$. Assume that the usual conditions $\left(F_{1}\right)$ to $\left(F_{4}\right)$ of $[\mathrm{CW}\}$ are satisfied.

A process $M=\left\{M_{z}, z \in \bar{T}\right\}$ is a martingale if $M_{z}$ is a real valued, integrable and $\mathcal{F}_{z}$-adapted random variable, and for any $z \leq z^{\prime} E\left(M_{z^{\prime}} / \mathcal{F}_{z}\right)=M_{z} . M$ is a strong martingale if $M$ vanishes on the axes and $E\left\{M\left(\left(z, z^{\prime}\right]\right) / \mathcal{F}_{z}^{1} \vee \mathcal{F}_{z}^{2}\right\}=0$, for each $z \leq z^{*}$.

For $p \geq 1, m_{c}^{p}$ will denote the class of all continuous martingales, vanishing on the axes, such that $E\left(\left|M_{z}\right|^{p}\right)<\infty$ for all $z \in \vec{T}$, and $m_{c, s}^{p}$ the subspace of strong martingales which are in $m_{c}^{p}$.

A subset of $T \times \Omega$ is called a predictable rectangle if it can be written as $\left(z, z^{\prime}\right] \times F$, with $F \in \mathcal{F}_{z}$. The set of all predictable rectangles will be denoted by $\mathcal{R}$, and the field generated by $\mathcal{R}$ will be $\mathcal{R}^{\prime}$. The $\sigma$-field $\mathcal{P}$, generated by $\mathcal{R}$ is called the predictable $\sigma$-field.

A process $h=\left(h_{z}\right)_{z \in T}$ is a predictable process if the map

$$
\begin{aligned}
T \times \Omega & \rightarrow \mathbb{R} \\
(z, \omega) & \rightarrow h(z, \omega)
\end{aligned}
$$


is $\mathcal{P}$-measurable (we consider in $\mathbb{R}$ the Borel $\sigma$-field $\mathcal{B}$ ). $\mathcal{H}_{b}(\mathcal{P})$ will be the space of all bounded predictable processes, and $\mathcal{E}$ the subspace of $\mathcal{H}_{b}(\mathcal{P})$ of the simple predictable processes, i.e.

$$
h=\sum_{i=1}^{r} \alpha_{i} 1_{A_{i} \times F_{i}} \text { where } r \in \mathbf{N}^{*}, \alpha_{i} \in \mathbf{R}, \text { and }
$$

$A_{i} \times F_{i} \in \mathcal{R} . J_{c}$ will be the set of all adapted continuous processes on $\bar{T}$. If $X \in J_{c}$ we can define the process $X^{*}$ as: $X_{z}^{*}=\sup _{z^{*} \leq z}\left|X_{z^{\prime}}\right|$ which also belongs to $J_{c}$ (and; in particular, $\left(X_{z}^{*}\right)_{z \in T}$ is predictable).

Consider the spaces of functions $L_{p}(P)=L_{p}(\Omega, \mathcal{F}, P), 0 \leq p<\infty$, with their usual topologies.

All constants will be written $C$, although they may vary from one expression to another one.

Definition 2.1. A $L_{p}(P)$-stochastic measure $\mu$ is an additive map defined on $\mathcal{R}^{\prime}$, taking values in $L_{p}(P)$ and satisfying:

(2.1.a) $\mu(A \times F)=1_{F} \mu(A \times F), \forall A \times F \in \mathcal{R}$.

(2.1.b) $\mu\left(R^{\prime}\right)$ is a bounded subset of $L_{p}(P)$.

(2.1.c) $\lim _{n}\left\|\mu\left(R_{n}\right)\right\|_{p}=0$ for any decreasing sequence of elements belonging to $R$ such that $\bigcap_{n} R_{n}=\phi$.

From this definition it follows that a stochastic measure $\mu$ has a unique $\sigma$ additive extension on $\mathcal{P}$, for the usual topology of $L_{p}(P)$. (See [MP]).

Remark. It is easy to see that (2.1.a) is equivalent to

$$
\mu(A \times F)=1_{F} \mu(A \times \Omega), \forall A \times F \in \mathcal{R} .
$$

We can associate to every process $M=\left(M_{z}\right)_{z \in T}$ a map satisfying (2.1.a) putting $\mu^{M}(\dot{A} \times F)=1_{F} M(A), \forall A \times F \in \mathcal{R}$.

This map can be extended to an additive map on $\mathcal{E}$ (and, consequently, on $\left.\mathcal{R}^{\prime}\right)$ :

If $h=\sum_{i=1}^{r} \alpha_{i} 1_{A_{i} \times F_{i}}$, then $\mu^{M}(h)=\sum_{i=1}^{r} \alpha_{i} 1_{F_{i}} M\left(A_{i}\right)$.

Furthermore if $M_{z} \in L_{p}(P)$ for any $z \in T$, then $\mu^{M}(\mathcal{E}) \subset L_{p}(P)$, but notice that $\mu^{M}$ may not verify (2.1.b) and (2.1.c).

Definition 2.2. Let $M$ be a process. If $\mu^{M}$ is a $L_{p}(P)$-stochastic measure we will say that the process $M$ defines a $L_{p}(P)$-stochastic measure.

We can define the stochastic integral with respect to a $L_{p}(P)$-stochastic measure $\mu$ for a class of processes that we will call $\mathcal{L}^{1}\left(\mu, L_{p}(P)\right) \supset \mathcal{H}_{b}(\mathcal{P})$. See [MP] and [B]. An important class of processes of $\mathcal{L}^{1}\left(\mu, L_{0}(P)\right)$ is given by the following proposition.

Proposition 2.3. If $\mu$ is a $L_{0}(P)$-stochastic measure, then any predictable process $h$ such that $\sup _{z \in T}|h(z)|$ is $\mathcal{F}$-measurable and finite $P$-a.s. belongs to $\mathcal{L}^{1}\left(\mu, L_{0}(P)\right)$. 
This extension of the integral with respect to a $L_{p}(P)$-stochatic measure satisfies the Dominated Convergence Theorem in the following sense:

Theorem 2.4. Let $\left(h_{n}\right)_{n}$ be a sequence of elements of $\mathcal{L}^{1}\left(\mu, L_{p}(P)\right)$ such that converges simply to $h$, and dominated by an element of $\mathcal{L}^{1}\left(\mu, L_{\mathrm{p}}(P)\right)$, it follows that $h \in \mathcal{L}^{1}\left(\mu, L_{p}(P)\right)$ and the seguence $\left(\mu\left(h_{n}\right)\right)_{n}$ converges to $\mu(h)$ in $L_{p}(P)$.

We point out that Bichteler defines a stochactic measure as an application defined in $\mathcal{R}^{\prime}$ taking values in $L_{p}(P)$ such that it has a unique $\sigma$-additive extension on $\mathcal{P}$ for the usual topology in $L_{p}(P)$ (see [B]). He also calls $L_{p}(P)$ integrator a process $M$ for which the integral defined for the simple predictable processes can be extended linearly and also with continuity to a vectorial space that contains the bounded predictable processes and such that this extension satisfies the dominated convegence theorem. We will follow the definition given in 2.1 .

Now we define the spaces of "semimartingales" related with the Itô's formula of $[\mathrm{A}]$.

From now on we shall denote by $\mu^{k}$ the additive map defined by the process $M^{k}$ (instead $\left.\mu^{M^{k}}\right)$.

Definition 2.5. A process $M \in J_{\mathrm{c}}$ is a $L_{p}(P)$-semimartingale of order $m$ $\left(m \in \mathbb{N}^{*}\right)$ if:

(2.5.1) $\forall k=1, \ldots, m$ the process $M^{k}$ defines a $L_{p}(P)$-stochastic measure denoted by $\mu^{k}$.

(2.5.2) $\forall k=1, \ldots, m$ the process $\left(M^{*}\right)^{m-k}$ is $\mu^{k}$-integrable.

\subsection{Notations.}

(2.6.1) $S_{c}^{m}\left(L_{p}(P)\right)$ is the set of elements of $J_{c}$ that are $L_{p}(P)$-semimartingales of order $m$.

(2.6.2) $S_{c}^{\infty}\left(L_{p}(P)\right)=\bigcap_{m} S_{c}^{m}\left(L_{p}(P)\right)$.

If $X \in \mathcal{L}^{1}\left(\mu, L_{p}(P)\right)$ we will also write $\int_{\left\{z, z^{\prime}\right\}} X d M$ instead of $\mu^{M}\left(1_{\left\{z, z^{\prime}\right\}} X\right)$.

For one-parameter processes the Dellacherie-Mokobodzki Theorem gives the equivalence between semimartingales and processes that define $L_{0}$ stochastic measures. Furthermore the measures that appear in the Itô's formula for semimartingales $X$ can be expressed in terms of the measures associated to the processes $X^{r}, r=1,2$.

In the two-parameter case, if we can establish an Itô's formula for certain stochastic measures $\mu^{(r)}$, these could be also expressed in terms of the stochastic measures associated to the powers of the semimartingale. More precisely, if $M$ is such that we have an Itô's formula

$$
\mu^{(f \circ M)}(h)=\sum_{r=1}^{m} \frac{1}{r^{!}} \mu^{(r)}\left(f^{(r)}(M) h\right), \quad \forall h \in \mathcal{H}_{b}(\mathcal{P}),
$$


putting $f(x)=x, x^{2}, x^{3}, \ldots, x^{m}$ we can see that the measures $\mu^{(r)}$ can be determined in terms of $\mu^{k}$ :

$$
\begin{array}{ll}
k=I, & \mu^{1}(h)=\mu^{(1)}(h), \\
k=2, & \mu^{2}(h)=2 \mu^{(1)}(M h)+\mu^{(2)}(h) \text { and so } \\
& \mu^{(2)}(h)=-2 \mu^{1}(M h)+\mu^{2}(h), \\
k=3, & \mu^{3}(h)=3 \mu^{(1)}\left(M^{2} h\right)+3 \mu^{(2)}(M h)+\mu^{(3)}(h) \quad \text { and so } \\
& \mu^{(3)}(h)=3 \mu^{1}\left(M^{2} h\right)-3 \mu^{2}(M h)+\mu^{3}(h) .
\end{array}
$$

By induction we can prove that we should obtain

$$
\mu^{(k)}(h)=\sum_{r=1}^{k}\left(\begin{array}{l}
k \\
r
\end{array}\right)(-I)^{k-r} \mu^{r}\left(M^{k-r} h\right) .
$$

Allain has defined the spaces of semimartingales in such a way that the measures $\mu^{(r)}$ are well defined. In fact we have the following Definition-Theorem:

Definition 2.7. If $M \in S_{c}^{m}\left(L_{p}(P)\right)$, there exist some $L_{p}(P)$-stochastic measures, called $\mu^{(k)}, k=1, \ldots, m$, defined by

$$
\mu^{(k)}(h)=\sum_{r=1}^{k}(-1)^{k-r}\left(\begin{array}{l}
k \\
r
\end{array}\right) \mu^{r}\left(h M^{k-r}\right), \quad h \in \mathcal{H}_{b}(\mathcal{P}) .
$$

Definition 2.8. If $M \in S_{c}^{m}\left(L_{p}(P)\right)$, for $k=1, \ldots, m$ we define the process $M^{(k)}$ in the following way:

$$
\begin{aligned}
& M_{z}^{(k)}=\mu^{(k)}\left(R_{z} \times \Omega\right) \quad \text { if } \quad z \in(0,1] \times(0,1] \\
& M_{z}^{(k)}=0 \quad \text { on the axes. }
\end{aligned}
$$

The processes $M^{(k)}$ define the measures $\mu^{(k)}, k=1, \ldots, m$.

We can now state the Allain's formula more precisely.

Itô's formula 2. If $M \in S_{c}^{\infty}\left(L_{0}(P)\right)$ is such that there is $m \in \mathbf{N}^{*}$ for which $\forall k \geq m+1, \mu^{(k)} \equiv 0$. Then, for any $f \in \mathcal{C}^{m}, f \circ M$ is also in $S_{c}^{\infty}\left(L_{0}(P)\right)$ and the stochastic measure associated to $f \circ M$ satisfies

$$
\mu^{f \circ M}(D)=\sum_{r=1}^{m} \frac{1}{r !} \mu^{(r)}\left(1_{D} f^{(r)} \circ M\right)
$$

for any predictäble set $D$.

In particular

$$
\Delta_{\left(z, z^{\prime}\right)} f \circ M=\sum_{r=1}^{r} \frac{1}{r !} \int_{\left(z, z^{\prime}\right]} f^{(r)}\left(M_{u}\right) d M_{u}^{(r)} .
$$


The proof is based on the fact that the conclusion of the Theorem is clear when we take $f(x)=x^{k}$, and then by linearity is also right when $f$ is a polynomial.

Then, the Theorem can be proved assuming that $M$ is bounded, by using that, in this case, $f \circ M$ and its derivatives up to order $m$ can be uniformly aproximated by $P_{n}(M)$. Finally the general case is studied by using several previous lemmas.

In order to prove II we will proceed as follows: for $M \in m_{\mathrm{c}}^{4}$ we consider the additive map associated to the process $\bar{M}^{(k)}: \bar{\mu}^{(k)}$ defined as $\bar{\mu}^{(k)}\left(1_{F} 1_{\left(z, z^{\prime}\right]}\right)=$ $1_{F} \bar{M}^{(k)}\left(\left(z, z^{\prime}\right]\right), \forall z<z^{\prime}, \forall F \in \mathcal{F}_{z}$. We will prove that $\bar{\mu}^{(k)}$ is an $L_{0}(P)$ stochastic measure which coincides with $\mu^{(k)}$, moreover, for all process $X \in J_{c}$

$$
\mu^{(r)}\left(1_{R_{x}} X\right)=\int_{R_{z}} X d M^{(r)}=\int_{R_{z}} X d \bar{M}^{(r)}
$$

where the integral on the left hand side is the integral with respect to a stochastic measure in the sense of Métivier-Pellaumail, [MP], and the other one is the integral defined by Sanz in (2).

As a consequence we will obtain that $\forall k \geq 5, \mu^{(k)} \equiv 0$ and therefore the total equivalence of the two Itô's formulas, when $M \in m_{c}^{\mathbf{4}}$.

\section{Proof of I}

In order to prove that $m_{c}^{4} \subset S_{c}^{\infty}\left(L_{0}(P)\right)$ we have to verify (2.5.1) and (2.5.2) for all $m \in \mathbf{N}^{*}$.

Once we have proved (2.5.1), (2.5.2) is obvious due to the continuity of $M$ and by Proposition 2.3 .

3.1: Now we prove that any $\mu^{k}$ satisfies (2.1.c), i.e. $\lim _{n}\left\|\mu^{k}\left(R_{n}\right)\right\|_{0}=0$ for any decreasing sequence $\left(R_{n}\right)_{n \in N}$ of elements of $\mathcal{R}$ such that $\cap R_{n}=\emptyset$. This fact is equivalent to show that the sequence of random variables $\left(\mu^{k}\left(R_{n}\right)\right)_{n}$ converges to 0 in probability.

Take $R_{n}=\left(z_{n}, z_{n}^{\prime}\right\} \times F_{n}$ with $F_{n} \in \mathcal{F}_{z_{n}}$ and $R_{n} \downarrow \emptyset$. Therefore $\mu^{k}\left(R_{n}\right)=$ $1_{F_{n}} M^{k}\left(\left(z_{n}, z_{n}^{\prime}\right)\right)$. Fix $\epsilon>0$, and consider the set $D_{a}=\left\{\omega: \sup _{z \in T}\left|M_{z}\right|<a\right\}$. Since $M$ is continuous there exists $a$ such that $P\left(D_{a}^{c}\right)<\epsilon$, and so

$$
P\left\{\left|1_{F_{n}} M^{k}\left(\left(z_{n}, z_{n}^{\prime}\right]\right)\right|>\lambda\right\}<\epsilon+P\left\{\left|1_{F_{n}}\left(M^{a}\right)^{k}\left(\left(z_{n}, z_{n}^{\prime}\right]\right)\right|>\lambda\right\}
$$

where $M^{a}(z)=(M(z) \wedge a) \vee(-a)$. By Chebyshev's inequality

$$
\begin{aligned}
& P\left\{\left|1_{F_{n}}\left(M^{a}\right)^{k}\left(\left(z_{n}, z_{n}^{\prime}\right]\right)\right|>\lambda\right\} \leq \lambda^{-1} E\left|1_{F_{n}}\left(M^{a}\right)^{k}\left(\left(z_{n}, z_{n}^{\prime}\right]\right)\right| \leq \\
& \lambda^{-j}\left(P\left(F_{n}\right)\right)^{1 / 2}\left(E\left(\left(M^{a}\right)^{k}\left(\left(z_{n}, z_{n}^{\prime}\right]\right)\right)^{2}\right)^{1 / 2}
\end{aligned}
$$


and this expression tends to zero when $n \rightarrow \infty$ since $(P \times \nu)\left(R_{n}\right) \downarrow 0(\nu$ is the Lebesgue measure in $\bar{T}$ ).

The condition (2.1.b) is given by the following proposition:

Proposition 3.2. If $M \in m_{c}^{4}$ then for any $k \in \mathbf{N} ; \mu^{k}\left(\mathcal{R}^{\prime}\right)$ is a bounded subset of $L_{0}(P)$.

Before giving the proof of this proposition we need some preliminar notations and results.

Notations. Any $A \in \mathcal{R}^{\prime}$ can be expressed as $A=\underset{\substack{\text { disjoint } \\ \text { dint }}}{\bigcup_{1}}\left\{\left(z_{h}, z_{h}^{\prime}\right) \times F_{h}\right\}$ where $F_{h} \in \mathcal{F}_{z_{h}}$. Without any loss of generality we can assume that $A=$ $\underset{(i, j) \in \alpha}{U}\left\{\left(z_{i j}, z_{i+1, j+1}\right] \times F_{i j}\right\}$ with $F_{i j} \in \mathcal{F}_{z_{i j}}$ and $\left\{z_{i j}\right\}_{i \in I, j \in J}$ is a finite partition of $T(I=\{0,1, \ldots, p\}, J=\{0,1, \ldots, q\})$ and $\alpha \subset I \times J$. Putting $F_{i j}=\emptyset$ when $(i, j) \notin \alpha$ we can write

$$
A=\bigcup_{(i, j) \in I \times J}\left\{\left(z_{i j}, \dot{z}_{i+1, j+1}\right] \times F_{i j}\right\}
$$

and so $\mu^{k}(A)=\sum_{i j} 1_{F_{i j}} M^{k}\left(\left(z_{i j}, z_{i+1, j+1}\right)\right)$. Let $u=z_{i j}=\left(s_{i}, t_{j}\right)$ be a point of the finite partition of $T$, we define $\bar{u}, \Delta u, \Delta_{u}^{1}$ and $\Delta_{u}^{2}$ as in the Introduction. Then we can prove the following lemma.

Lemma 3.3. (a) For all $\Delta_{u}=(u, \bar{u}]$ and for any $\ell \in \mathbf{N}$ we can express $M^{\ell}\left(\Delta_{u}\right)$ as $M^{\ell}\left(\Delta_{u}\right)=\sum_{j=1}^{\ell}\left(\begin{array}{l}\ell \\ j\end{array}\right) M_{u}^{\ell-j} \Delta_{u}^{j}(M)$ where

$$
\Delta_{u}^{j}(M)=\left(M_{\bar{u}}-M_{u}\right)^{j}-\left(M_{u \otimes \bar{u}}-M_{u}\right)^{j}-\left(M_{\bar{u} \otimes u}-M_{u}\right)^{j} .
$$

As a consequence of (a) we obtain

(b) $\Delta_{v}^{k}(M)=\sum_{r=1}^{k}\left(\begin{array}{l}k \\ r\end{array}\right)(-1)^{k-r} M_{u}^{k-r} M^{r}\left(\Delta_{u}\right)$.

Proof: Part (a) follows directly from Newton's binomial.

$$
M_{v}^{\ell}=\sum_{j=0}^{\ell}\left(\begin{array}{l}
\ell \\
j
\end{array}\right) M_{u}^{\ell-j}\left(M_{v}-M_{u}\right)^{j}
$$

By using this relation with $v=u \otimes \bar{u}, \bar{u} \otimes u, \bar{u}$ we obtain

$$
M^{\ell}\left(\Delta_{u}\right)=M_{u}^{\ell}+M_{\bar{u}}^{\ell}-M_{u \otimes \bar{u}}^{\ell}-M_{\tilde{u} \otimes u}^{\ell}=\sum_{j=1}^{\ell}\left(\begin{array}{l}
\ell \\
j
\end{array}\right) M_{u}^{\ell-j} \Delta_{u}^{j}(M) .
$$


Part (b) follows from (a) by using the following binomial inversion formula : If $\forall \ell \leq n \quad$ (or $\forall \ell \in \mathbf{N}$ ) we have

$$
a_{\ell}=\sum_{j=1}^{\ell}\left(\begin{array}{l}
\ell \\
j
\end{array}\right) M^{\ell-j} b_{j}
$$

then $\forall k \leq n \quad$ (or $\forall k \in \mathbf{N}$ ) it holds that

$$
b_{k}=\sum_{r=1}^{k}\left(\begin{array}{l}
k \\
r
\end{array}\right)(-1)^{k-r} M^{k-r} a_{r} .
$$

Proof of Proposition 3.2: We must prove that for any sequence $\left(A_{n}\right)_{n}, A_{n} \in$ $\mathcal{R}^{\prime}$ and $\left(\alpha_{n}\right)_{n}, \alpha_{n} \in \mathbf{R}$ with $\lim _{n} \alpha_{n}=0$ we have that $P-\lim _{n} \alpha_{n} \mu^{k}\left(A_{n}\right)=0$, i.e. for all $\epsilon>0 \lim _{n} P\left\{\left|\alpha_{n} \mu^{k}\left(A_{n}\right)\right|>\epsilon\right\}=0$. Obviously this last property is equivalent to the following one: For every sequence $\left(K_{n}\right)_{n}$ of real numbers tending to $+\infty, \lim _{n} P\left\{\left|\mu^{k}\left(A_{n}\right)\right|>K_{n}\right\}=0$. Let $S^{n}$ be the partition associated with $A_{n}$.

So, we want to prove that $\lim _{n} P\left\{\left|\sum_{u \in S^{n}} 1_{F_{u}} M^{k}\left(\Delta_{u}\right)\right|>K_{n}\right\}=0$, and by Lemma 3.3 it is enough to show that for all $X \in J_{c}$ and for all $\ell \in \mathbb{N}$ $\lim _{n} P\left\{\left|\sum_{u \in S^{n}} 1_{F_{u}} X_{u} \Delta_{u}^{\ell}(M)\right|>K_{n}\right\}=0$. In the sequel we omit $u \in S^{n}$. Since

$$
\begin{aligned}
& \sum_{u} 1_{F_{u}} X_{u} \Delta_{u}^{\ell}(M)= \\
& \sum_{u} 1_{F_{u}} X_{u}\left[\left(M\left(\Delta_{u}\right)+M\left(\Delta_{u}^{1}\right)+M\left(\Delta_{u}^{2}\right)\right)^{\ell}-M\left(\Delta_{u}^{1}\right)^{\ell}-M\left(\Delta_{u}^{2}\right)^{\ell}\right]= \\
& \sum_{u} \sum_{m=0}^{\ell} \sum_{r=0}^{\ell-m}\left(\begin{array}{c}
\ell \\
m
\end{array}\right)\left(\begin{array}{c}
\ell-m \\
r
\end{array}\right) 1_{F_{u}} X_{u} M\left(\Delta_{u}\right)^{m} M\left(\Delta_{u}^{1}\right)^{r} M\left(\Delta_{u}^{2}\right)^{\ell-m-r}- \\
& \sum_{u} 1_{F_{u}} X_{u} M\left(\Delta_{u}^{1}\right)^{\ell}-\sum_{u} 1_{F_{u}} X_{u} M\left(\Delta_{u}^{2}\right)^{\ell},
\end{aligned}
$$

in order to prove the proposition it will be sufficient to show the following assertion:

$$
\lim _{n} P\left\{\left|\sum_{u} 1_{F_{u}} X_{u} M\left(\Delta_{u}\right)^{m} M\left(\Delta_{u}^{1}\right)^{r} M\left(\Delta_{u}^{2}\right)^{\ell-m-r}\right|>K_{n}\right\}=0
$$

where $\ell \in \mathbf{N} ; m=0,1, \ldots, \ell ; r=0,1, \ldots, \ell-m$, and when $m=0, r(\ell-m-r) \neq$ 0 .

Fix $\epsilon>0$. Set $D_{a}=\left\{\omega: \sup _{z \in T}\left|X_{z}(\omega)\right| \leq a, \sup _{z \in T}\left|M_{z}(\omega)\right| \leq a\right\}$. Since $X$ and $M$ are continuous, there exists $a>0$ such that $P\left(D_{a}^{\mathrm{c}}\right)<\epsilon$. Set $X^{a}=(X \wedge a) \vee(-a)$, in the same way we define $M^{a}$.

We consider all the possible cases: 1) $m, r, \ell-m-r>0$; 2) $r=0, m(\ell-m) \neq$ 0 ;3) $m=0, r(\ell-r) \neq 0$ and 4) $r=\ell-m=0$. 
I) $m, r, \ell-m-r>0$.

$$
\begin{aligned}
& P\left\{\left|\sum_{u} 1_{F_{v}} X_{u} M\left(\Delta_{u}\right)^{m} M\left(\Delta_{u}^{1}\right)^{r} M\left(\Delta_{u}^{2}\right)^{\ell-m-r}\right|>K_{n}\right\} \leq P\left(D_{a}^{c}\right)+ \\
& P\left\{\left|\sum_{u} 1_{F_{u}} X_{u}^{a} M^{a}\left(\Delta_{u}\right)^{m-1} M^{a}\left(\Delta_{u}^{1}\right)^{r-1} M^{a}\left(\Delta_{u}^{2}\right)^{\ell-m-r-1} M\left(\Delta_{u}\right) M\left(\Delta_{u}^{1}\right) M\left(\Delta_{u}^{2}\right)\right|>\right. \\
& \left.K_{n}\right\} \leq \epsilon+K_{n}^{-1} E\left(\sup _{u}\left|X_{u}^{a} M^{a}\left(\Delta_{u}\right)^{m-1} M^{a}\left(\Delta_{u}^{1}\right)^{r-1} M^{a}\left(\Delta_{u}^{2}\right)^{\ell-m-r-1}\right|\right. \\
& \left.\sum_{u}\left|M\left(\Delta_{u}\right) M\left(\Delta_{u}^{1}\right) M\left(\Delta_{u}^{2}\right)\right|\right) \leq \epsilon+C K_{n}^{-1} E\left(\sum_{u}\left|M\left(\Delta_{u}\right) M\left(\Delta_{u}^{1}\right) M\left(\Delta_{u}^{2}\right)\right|\right) .
\end{aligned}
$$

In order to prove that this expression tends to zero it suffices to establish that $E\left(\sum_{v}\left|M\left(\Delta_{u}\right) M\left(\Delta_{u}^{ \pm}\right) M\left(\Delta_{u}^{2}\right)\right|\right)$ is bounded by a constant which does not depend on the partition that we have considered.

$$
\begin{aligned}
& E\left(\sum_{u}\left|M\left(\Delta_{u}\right) M\left(\Delta_{u}^{1}\right) M\left(\Delta_{u}^{2}\right)\right|\right) \leq \\
& E\left[\left(\sum_{u} M\left(\Delta_{u}\right)^{2}\right)^{1 / 2}\left(\sum_{u} M\left(\Delta_{u}^{1}\right)^{2} M\left(\Delta_{u}^{2}\right)^{2}\right)^{1 / 2}\right] \leq \\
& \left\{E\left(\sum_{u} M\left(\Delta_{u}\right)^{2}\right) E\left(\sum_{u} M\left(\Delta_{u}^{1}\right)^{2} M\left(\Delta_{u}^{2}\right)^{2}\right)\right\}^{1 / 2}
\end{aligned}
$$

By Burkholder's inequality the first factor of the later product is bounded by $C E M_{11}^{2}$. Now we study the second one:

$$
\begin{aligned}
& E\left(\sum_{u} M\left(\Delta_{u}^{1}\right)^{2} M\left(\Delta_{u}^{2}\right)^{2}\right) \leq E\left(\sum_{j}\left(\sup _{i} M\left(\Delta_{u}^{2}\right)^{2} \sum_{i} M\left(\Delta_{u}^{1}\right)\right)\right) \leq \\
& E\left(\sup _{j} \sum_{i} M\left(\Delta_{u}^{1}\right)^{2} \sum_{j} \sup _{i} M\left(\Delta_{u}^{2}\right)^{2}\right) \leq \\
& \left\{E\left(\sum_{i} \sup _{j} M\left(\Delta_{u}^{1}\right)^{2}\right)^{2} E\left(\sum_{j} \sup _{i} M\left(\Delta_{u}^{2}\right)^{2}\right)^{2}\right\}^{1 / 2} .
\end{aligned}
$$

Clearly it is enough to prove that the first expectation is bounded.

Consider the increasing, continuous and $\mathcal{F}_{1 t}$-adapted process defined by $A_{i}=\sum_{i} \sup _{t} \leq t\left(M\left(s_{i+1}, \tau\right)-M\left(s_{i}, r\right)\right)^{2}$. We have that $E\left(\sum_{i} \sup _{j} M\left(\Delta_{t}^{1}\right)^{2}\right)^{2} \leq E\left(A_{1}^{2}\right)$. Find the potential associated to $A_{t}$,

$$
\begin{aligned}
& Z_{t}=E\left(A_{1}-A_{t} / \mathcal{F}_{1 t}\right)= \\
& E\left(\sum _ { i } \left(\sup _{\tau \leq 1}\left(M\left(s_{i+1}, \tau\right)-M\left(s_{i}, \tau\right)\right)^{2}\right.\right. \\
& \left.\left.-\sup _{\tau \leq t}\left(M\left(s_{i+1}, \tau\right)-M\left(s_{i}, \tau\right)\right)^{2}\right) / \mathcal{F}_{1 t}\right) \leq \\
& E\left(\sum_{i} \sup _{\tau \geq t}\left(M\left(s_{i+1}, \tau\right)-M\left(s_{i}, \tau\right)\right)^{2} / \mathcal{F}_{1 t}\right) \leq \\
& C \sum_{i} E\left(\left(M s_{i+1}-M_{s_{i}, 1}\right)^{2} / \mathcal{F}_{1 t}\right)=: m_{t},
\end{aligned}
$$


where in the last inequality we have applied the Doob's inequality for conditioned expectations. Since $m_{t}$ is a $\mathcal{F}_{1, t}$-martingale, by Garsia - Neveu's inequality

$$
E\left(A_{1}^{2}\right) \leq C E\left(m_{1}^{2}\right)=C E\left(\sum_{i}\left(M_{s_{i+1}, 1}-M_{s_{i}, 1}\right)^{2}\right)^{2}
$$

By Burkholder's inequality applied to the discrete parameter martingale $\left\{M_{s_{i}, 1}, i=1, \ldots p_{n}\right\}$ the last expression is bounded by $C E\left(M_{11}^{4}\right)$. Hence we have concluded case 1 .

2) $r=0, m(\ell-m) \neq 0$.

We distinguish two new cases; $m \geq 2$ and $m=1$.

2.a) $m \geq 2$.

$$
\begin{aligned}
& P\left\{\left|\sum_{u} 1_{F_{u}} X_{u} M\left(\Delta_{u}\right)^{m} M\left(\Delta_{u}^{2}\right)^{\ell-m}\right|>K_{n}\right\} \leq \\
& P\left(D_{a}^{c}\right)+P\left\{\left|\sum_{u} I_{F_{u}} X_{u}^{a} M^{a}\left(\Delta_{u}\right)^{m-2} M^{a}\left(\Delta_{u}^{2}\right)^{\ell-m} M\left(\Delta_{u}\right)^{2}\right|>K_{n}\right\} \leq \\
& \epsilon+C K_{n}^{-1} E\left(\sum_{u} M\left(\Delta_{u}\right)^{2}\right) \leq \epsilon+C K_{n}^{-1} E\left(M_{11}^{2}\right) .
\end{aligned}
$$

2.b) $m=1$.

The relation $\ell-m \neq 0$ implies $\ell \geq 2$, and then

$$
\begin{aligned}
& P\left\{\left|\sum_{u} 1_{F_{u}} X_{u} M\left(\Delta_{u}\right) M\left(\Delta_{u}^{2}\right)^{\ell-1}\right|>K_{n}\right\} \leq \\
& P\left(D_{a}^{c}\right)+P\left\{\left|\sum_{u} 1_{F_{u}} X_{u}^{a} M^{a}\left(\Delta_{u}^{2}\right)^{\ell-2} M\left(\Delta_{u}\right) M\left(\Delta_{u}^{2}\right)\right|>K_{n}\right\} \leq \\
& \epsilon+K_{n}^{-2} E\left(\sum_{u} 1_{F_{u}} X_{u}^{a} M^{a}\left(\Delta_{u}^{2}\right)^{\ell-2} M\left(\Delta_{u}\right) M\left(\Delta_{u}^{2}\right)\right)^{2} .
\end{aligned}
$$

By Burkhoider's inequality applied to the $\mathcal{F}_{s_{h}, 1}$-martingale $\sum_{i=0}^{h-1} \sum_{j} 1_{F_{i j}} X_{z_{i j}}^{a} M\left(\Delta_{z_{i j}}\right) M\left(\Delta_{z_{i j}}^{2}\right) M^{a}\left(\Delta_{z_{i j}}^{2}\right)^{\ell-2}$, the last expression can be bounded by:

$$
\begin{aligned}
& \epsilon+C K_{n}^{-2} E\left[\sum_{i}\left(\sum_{j} 1_{F_{u}} X_{u}^{a} M^{a}\left(\Delta_{u}^{2}\right)^{\ell-2} M\left(\Delta_{u}\right) M\left(\Delta_{u}^{2}\right)\right)^{2}\right] \leq \\
& \epsilon+C K_{n}^{-2} E\left[\sum_{i}\left(\sum_{j} 1_{F_{u}}\left(X_{u}^{a}\right)^{2} M^{a}\left(\Delta_{u}^{2}\right)^{2 \ell-4} M\left(\Delta_{u}\right)^{2}\right) \cdot\left(\sum_{j} M\left(\Delta_{u}^{2}\right)^{2}\right)\right] \leq \\
& \epsilon+C K_{n}^{-2} E\left(\sup _{i}\left(\sum_{j} M\left(\Delta_{u}^{2}\right)^{2}\right) \cdot\left(\sum_{u} M\left(\Delta_{u}\right)^{2}\right)\right) \leq
\end{aligned}
$$

$$
\epsilon+C K_{n}^{-2}\left\{E\left(\sum_{j} \sup _{i} M\left(\Delta_{u}^{2}\right)^{2}\right)^{2} E\left(\sum_{u} M\left(\Delta_{u}\right)^{2}\right)^{2}\right\}^{1 / 2} .
$$


At the end of case I we have seen that $E\left(\sum_{j} \sup _{i} M\left(\Delta_{u}^{2}\right)^{2}\right)^{2} \leq C E\left(M_{11}^{4}\right)$ and, by Burkholder's inequality applied to the $\left\{\mathcal{F}_{s_{n}, t_{k}}, h=1, \ldots, p_{n} ; k=1, \ldots, q_{n}\right\}$ martingale $\sum_{j=1}^{k-1} \sum_{i=0}^{k-1} M\left(\Delta_{u}\right)$,

$E\left(\sum_{i, j} M\left(\Delta_{u}\right)^{2}\right)^{2} \leq C E M_{11}^{4}$, then (3) is bounded by $\epsilon+C K_{n}^{-2} E M_{11}^{4}$.

3) $m=0, r(\ell-r) \neq 0$.

3.a) $r \geq 2, \ell-r \geq 2$.

$$
\begin{aligned}
& P\left\{\left|\sum_{u} 1_{F_{u}} X_{u} M\left(\Delta_{u}^{1}\right)^{r} M\left(\Delta_{u}^{2}\right)^{\ell-r}\right|>K_{n}\right\} \leq \\
& P\left(D_{u}^{c}\right)+P\left\{\left|\sum_{u} 1_{F_{u}} X_{u}^{a} M^{a}\left(\Delta_{u}^{1}\right)^{r-2} M^{a}\left(\Delta_{u}^{2}\right)^{\ell-r-2} M\left(\Delta_{u}^{1}\right)^{2} M\left(\Delta_{u}^{2}\right)^{2}\right|>K_{n}\right\} \leq \\
& \epsilon+K_{n}^{-1} E\left(\sum_{u}\left|1_{F_{u}} X_{u}^{a} M^{a}\left(\Delta_{u}^{1}\right)^{r-2} M^{a}\left(\Delta_{u}^{2}\right)^{\ell-r-2}\right| M\left(\Delta_{u}^{1}\right)^{2} M\left(\Delta_{u}^{2}\right)^{2}\right) \leq \\
& \epsilon+C K_{n}^{-1} E\left(\sum_{u} M\left(\Delta_{u}^{1}\right)^{2} M\left(\Delta_{u}^{2}\right)^{2}\right),
\end{aligned}
$$

and the last expectation has been studied in case 1.

3.b) $\ell-r=1, r=1$.

$$
\begin{aligned}
& P\left\{\left|\sum_{u} 1_{F_{u}} X_{u} M\left(\Delta_{u}^{1}\right) M\left(\Delta_{u}^{2}\right)\right|>K_{n}\right\} \leq \\
& P\left(D_{a}^{c}\right)+P\left\{\left|\sum_{u} 1_{F_{u}} X_{u}^{a} M\left(\Delta_{u}^{1}\right) M\left(\Delta_{u}^{2}\right)\right|>K_{n}\right\} \leq \\
& \epsilon+C K_{n}^{-2} E\left(\sum_{u} 1_{F_{u}} X_{u}^{a} M\left(\Delta_{u}^{1}\right) M\left(\Delta_{u}^{2}\right)\right)^{2}
\end{aligned}
$$

By applying Burkholder's inequality to the $\left\{\mathcal{F}_{s_{h}, t_{k}}, h=1, \ldots, p_{n} ; k=1, \ldots, q_{n}\right\}-$ martingale $\sum_{j=0}^{k-1} \sum_{i=0}^{h-1} 1_{F_{u}} X_{u}^{a} M\left(\Delta_{u}^{1}\right) M\left(\Delta_{u}^{2}\right)$, the last term is bounded by $\epsilon+C K_{n}^{-2} E\left(\sum_{u} M\left(\Delta_{u}^{1}\right)^{2} M\left(\Delta_{u}^{2}\right)^{2}\right)$ and it is similar as 3.a.

3.c) $\ell-r=1, r \geq 2$.

$$
\begin{aligned}
& P\left\{\left|\sum_{u} 1_{F_{u}} X_{u} M\left(\Delta_{u}^{1}\right)^{r} M\left(\Delta_{u}^{2}\right)\right|>K_{n}\right\} \leq \\
& P\left(D_{u}^{c}\right)+P\left\{\left|\sum_{u} 1_{F_{u}} X_{u}^{a} M^{a}\left(\Delta_{u}^{1}\right)^{r-2} M\left(\Delta_{u}^{1}\right)^{2} M\left(\Delta_{u}^{2}\right)\right|>K_{n}\right\} \leq \\
& \epsilon+K_{n}^{-1} E\left(\sum_{i}\left|\sum_{j} 1_{F_{u}} X_{u}^{a} M^{a}\left(\Delta_{u}^{1}\right)^{r-2} M\left(\Delta_{u}^{1}\right)^{2} M\left(\Delta_{u}^{2}\right)\right|\right) \leq \\
& \epsilon+C K_{n}^{-1} E\left(\sum_{i}\left(\sum_{j} 1_{F_{u}}\left(X_{u}^{a}\right)^{2} M^{a}\left(\Delta_{u}^{1}\right)^{2 r-4} M\left(\Delta_{u}^{1}\right)^{4} M\left(\Delta_{u}^{2}\right)^{2}\right)^{1 / 2}\right) \leq \\
& \epsilon+C K_{n}^{-1} E\left(\sum_{i}\left(\sum_{j} M\left(\Delta_{u}^{1}\right)^{4} M\left(\Delta_{u}^{2}\right)^{2}\right)^{1 / 2}\right) .
\end{aligned}
$$


Where we have applied Davis inequality to the $\left\{\mathcal{F}_{1, t_{k}}, k=1, \ldots, q_{n}\right\}$-martingale

$$
\sum_{j=0}^{k-1} 1_{F_{u}} X_{u}^{a} M^{a}\left(\Delta_{u}^{1}\right)^{r-2} M\left(\Delta_{u}^{1}\right)^{2} M\left(\Delta_{u}^{2}\right) .
$$

Next, we will study the last expectation.

$$
\begin{aligned}
& \left.E\left(\sum_{i}\left(\sum_{j} M\left(\Delta_{u}^{1}\right)^{4} M\left(\Delta_{u}^{2}\right)^{2}\right)^{1 / 2}\right) \leq\left(E \sum_{i} \sup _{j}\left|M\left(\Delta_{u}^{1}\right)\right| \sum_{j} M\left(\Delta_{u}^{2}\right)^{2} M\left(\Delta_{u}^{1}\right)^{2}\right)^{1 / 2}\right) \leq \\
& E\left[\left(\sum_{i} \sup _{j} M\left(\Delta_{u}^{1}\right)^{2}\right)^{1 / 2}\left(\sum_{u} M\left(\Delta_{u}^{2}\right)^{2} M\left(\Delta_{u}^{1}\right)^{2}\right)^{1 / 2}\right] \leq \\
& \left\{E\left[\sum_{i} \sup _{j} M\left(\Delta_{u}^{1}\right)^{2}\right] E\left[\sum_{u} M\left(\Delta_{u}^{2}\right)^{2} M\left(\Delta_{u}^{1}\right)^{2}\right]\right\}^{1 / 2} .
\end{aligned}
$$

Observe that $M\left(\Delta_{u}^{1}\right)^{2}=\left(M_{s_{i+1}, t_{j}}-M_{s_{i}, t_{j}}\right)^{2}$ and applying Doob's inequality to the $\mathcal{F}_{1, t_{j}}$-martingale $M_{s_{i+1}, t_{j}}-M_{s_{i}, t_{j}}$ we have that

$$
E\left[\sum_{i} \sup _{j} M\left(\Delta_{u}^{I}\right)^{2}\right] \leq C E\left(\sum_{i}\left(M_{s_{i+1}, 1}-M_{s_{i, 1}}\right)^{2}\right) \leq C E M_{11}^{2} .
$$

The term $E\left[\sum_{u} M\left(\Delta_{\mathfrak{u}}^{2}\right)^{2} M\left(\Delta_{\mathfrak{u}}^{1}\right)^{2}\right]$ has been considered in case 1 .

4) $r=\ell-m=0$.

4.a) $m=1$.

$$
P\left\{\left|\sum_{u} 1_{F_{u}} X_{u} M\left(\Delta_{u}\right)\right|>K_{n}\right\} \leq \epsilon+K_{n}^{-2} E\left(\sum_{u} 1_{F_{u}} X_{u}^{a} M\left(\Delta_{u}\right)\right)^{2} .
$$

By Burkholder's inequality for discrete two-parameter martingales

$$
E\left(\sum_{u} 1_{F_{u}} X_{u}^{a} M\left(\Delta_{u}\right)\right)^{2} \leq C E\left(\sum_{u} M\left(\Delta_{u}\right)^{2}\right) \leq C E M_{11}^{2} .
$$

4.b) $r=\ell-m=0, m \geq 2$.

$$
\begin{aligned}
& P\left\{\left|\sum_{u} 1_{F_{u}} X_{u} M\left(\Delta_{u}\right)^{m}\right|>K_{n}\right\} \leq \\
& \epsilon+K_{n}^{-1} E\left(\sum_{u} 1_{F_{u}}\left|X_{u}^{a} M^{a}\left(\Delta_{u}\right)^{m-2}\right| M\left(\Delta_{u}\right)^{2}\right) \leq \\
& \epsilon+C K_{n}^{-1} E\left(\sum_{u} M\left(\Delta_{u}\right)^{2}\right) \leq \epsilon+C K_{n}^{-1} E M_{11}^{2},
\end{aligned}
$$

This ends the proof of the proposition. 


\section{Proof of II}

Lemma 4.1. Let $\mu$ be a $L_{0}(P)$ stochastic measure and

$$
h=\sum_{i=r}^{r} \alpha_{i} l_{\left(z_{i}, z_{i}^{\prime}\right)}
$$

where $\alpha_{i}$ is a $\mathcal{F}_{z_{i}}$-measurable and a a.s. finite random variable, $i=1, \ldots, r$. (In particular $\sup _{\mathrm{z} \in T}|h(z)|$ is $\mathcal{F}$-measurable and a a.s. finite random variable, and so $h \in L^{1}\left(\mu, L_{0}(P)\right)$. Then the stochastic integral of $h$ with respect to $\mu$ can be obtained by

$$
\mu(h)=\sum_{i=r}^{r} \alpha_{i} \mu\left(1_{\left(z_{i}, z_{i}^{\prime}\right] \times \Omega}\right)
$$

Proof: By linearity of the integral with respect to stochastic measures we can assume that $h=\alpha 1_{\left(z, z^{\prime}\right)}$ where $\alpha$ is $\mathcal{F}_{z}$-measurable.

Then $\alpha$ will be the pointwise limit of simple functions of the form $\alpha^{(n)}=$ $\sum_{j=1}^{J_{n}} \beta_{n}^{j} I_{F_{n}}$ where $F_{n}^{j} \in \mathcal{F}_{z}$ and $\left\{F_{n}^{j}, j=1, \ldots, J_{n}\right\}$ form a partition of $\Omega$.

Hence $\forall u \in T$ we have that $h_{u}=\lim _{n \rightarrow \infty} \sum_{j=1}^{J_{n}} \beta_{n}^{j} 1_{F_{n}^{j} 1_{\left(z, z^{\prime}\right\}}}(u)$ a.s.. So if we can prove that

$$
\mu\left(\sum_{j=1}^{J_{n}} \beta_{n}^{j} 1_{F_{n}^{j}} 1_{\left(z, z^{\prime}\right]}\right)=\sum_{j=1}^{J_{n}} \beta_{n}^{j} \mu\left(1_{\left(z, z^{\prime}\right) \times F_{n}^{j}}\right)
$$

tends in probability to $\alpha \mu\left(1_{\left(z, z^{\prime}\right] \times \Omega}\right)$, from theorem 2.4 plus the uniqueness of the limit we will have that $\mu(h)=\alpha \mu\left(1_{\left(z, z^{\prime}\right] \times \Omega}\right)$.

Consider $\varepsilon>0$, then

$$
\begin{aligned}
& P\left\{\left|\sum_{j=1}^{J_{n}} \beta_{n}^{j} \mu\left(1_{\left(z, z^{\prime}\right] \times F_{n}^{j}}\right)-\alpha \mu\left(1_{\left(z, z^{\prime}\right] \times \Omega}\right)\right|>\varepsilon\right\}= \\
& \left.P\left\{\mid \sum_{j=1}^{J_{n}}\left(\beta_{n}^{j}-\alpha\right) 1_{F_{n}^{j}} \mu\left(1_{\left(z, z^{\prime}\right] \times F_{n}^{j}}\right)\right) \mid>\varepsilon\right\} \leq \\
& P\left\{\sup _{F \in \mathcal{F}_{z}}\left|\mu\left(1_{\left(z, z^{\prime}\right] \times F}\right)\right|\left(\sum_{j=1}^{J_{n}}\left|\beta_{n}^{j}-\alpha\right| 1_{F_{n}^{j}}\right)>\varepsilon\right\} .
\end{aligned}
$$

And since $\sup _{F \in \mathcal{F}_{z}}\left|\mu\left(1_{\left(z, z^{\prime}\right) \times F}\right)\right|=\sup _{F \in F_{z}}\left|\mu\left(1_{\left(z, z^{\prime}\right] \times \Omega}\right) 1_{F}\right|=\left|\mu\left(1_{\left(z, z^{\prime}\right] \times \Omega}\right)\right|$ the last term is measurable and its probability is less or equal than

$$
P\left\{\left|\mu\left(1_{\left(z, z^{\prime}\right\} \times \Omega}\right)\right|>K\right\}+P\left\{\left|\sum_{j=1}^{J_{n}}\right| \beta_{n}^{j} 1_{F_{n}^{j}}-\alpha 1_{F_{n}^{j}} \mid>\frac{\varepsilon}{K}\right\} \quad, \forall K>0 .
$$


Fixed $\delta>0$, since $\mu\left(1_{\left(z, z^{\prime}\right] \times \Omega}\right) \in L_{0}(P)$, and in particular is a.s. finite, there exists $K$ such that the first term of this last expression can be considered less that $\delta / 2$. For this value of $K$, there exists $n_{0}$ such that $\forall n \geq n_{0}$ the second term can be also considered less than $\delta / 2$. This fact proves the lemma.

Remark. The same result also holds if $\mu$ is a $L_{p}(P)$-stochastic measure and if the $\alpha_{i} \in L_{\infty}(P)$ and are $\mathcal{F}_{z_{i}}$-measurables. The proof is similar since if $\alpha$ is a $\mathcal{F}_{z}$-measurable random variable and belongs to $L_{\infty}$, then it is a uniform limit of simple functions, except perhaps in a null set.

Proposition 4.2. Let $M \in m_{c}^{4}$. Consider the additive map associated to the process $\bar{M}^{(k)}$ defined on $\mathcal{E}$ :

$$
\bar{\mu}^{(k)}\left(1_{F \times\left(z, z^{\prime}\right]}\right)=1_{F} \bar{M}^{(k)}\left(\left(z, z^{\prime}\right]\right)=1_{F} P-\lim _{\left|S^{n}\right| \downarrow 0} \sum_{u \in S^{n} \cap\left[z, z^{\prime}\right)} \Delta_{u}^{k}(M) .
$$

Then

(a) $\bar{\mu}^{(k)}$ is a $L_{0}(P)$-stochastic measure and coincides with $\mu^{(k)}$.

(b) For all $X \in J_{c}$ the stochastic integral in the [MP] sense, with respect to the stochastic measure $\mu^{(k)}$ can be computed as

$$
\int_{R_{x}} X d M^{(k)}=\int_{R_{x}} X d \bar{M}^{(k)} \quad \forall k \in \mathbf{N}
$$

Proof: (a) Since $M \in m_{\mathrm{c}}^{4} \subset S_{c}^{\infty}\left(L_{0}(P)\right)$, it suffices to see that

$$
\left.\bar{\mu}^{(k)}\left(1_{\left(z, z^{\prime}\right]} 1_{F}\right)=\mu^{(k)}\left(1_{\left(z, z^{\prime}\right]}\right]_{F}\right) \quad \forall z<z^{\prime} \quad \forall F \in \mathcal{F}_{z},
$$

or equivalently

$$
\mu^{(k)}\left(1_{\left\langle z, z^{\prime}\right] \times F}\right)=1_{F} P-\lim _{\left|S^{n}\right| 10} \sum_{t \in S^{n} \cap\left[z, z^{\prime}\right)} \Delta_{u}^{k}(M) .
$$

Observe that (a) is a consequence of (b), because

$$
\mu^{(k)}\left(1_{\left(z, z^{\prime}\right]} X\right)=P-\lim _{n} \sum_{u \in S^{n} \cap\left[z, z^{\prime}\right)} X_{u} \Delta_{u}^{k}(M),
$$

if we take $X=1_{\Omega}$. (We use that $\mu^{(k)}\left(1_{\left(z, z^{\prime}\right] \times F}\right)=1_{F} \mu^{(k)}\left(1_{\left(z, z^{t}\right] \times \Omega}\right.$ ) for all $F \in \mathcal{F}_{z}$ ).

Note that in order to prove (b) we must show that if $M \in m_{\mathrm{c}}^{4}$ (and then $\left.M \in S_{c}^{m}\left(L_{0}(P)\right), \forall m \in \mathbf{N}\right)$

$$
\mu^{(k)}\left(1_{R_{z}} X\right)=P-\lim _{n} \sum_{u \in S_{z}^{n}} X_{u} \Delta_{\dot{u}}^{k}(M)
$$


where $S^{n}$ is a sequence of partitions of $\bar{T}$ such that $\left|S^{n}\right| \downarrow 0$.

Lemma 4.9 in [A] proves the same result for $M \in S_{c}^{\infty}\left(L_{p}(P)\right)$ for all $p \geq 0$, and $X$ equal to $f(M)$ and $f$ continuous and bounded. In our case $p=0$ and $X$ is any process belonging to $J_{C}$ but the proof is essentially the same.

Next, we prove (4). Let $\left(S^{n}\right)_{n}$ be a sequence of partitions of $\bar{T}$ such that $\left|S^{n}\right| \downarrow 0$. By definition

$$
\mu^{(k)}\left(1_{R_{z}} X\right)=\sum_{r=1}^{k}(-1)^{k-r}\left(\begin{array}{l}
k \\
r
\end{array}\right) \mu^{r}\left(1_{R_{z}} X M^{k-r}\right)
$$

Consider the sequence of predictable processes

$$
\begin{aligned}
& X_{r}^{(n)}(v, \omega)=\sum_{u \in S^{n}} 1_{R_{z}}(v)\left(X_{u} M_{u}^{k-r}\right)(\omega) 1_{(u, \bar{u}]}(v) \\
& =\left(\sum_{u \in S_{x}^{n}} X_{u} M_{u}^{k-r} 1_{(u, \bar{u}]}\right) 1_{R_{x}}
\end{aligned}
$$

which tends simply to $1_{R_{z}} X M^{k-r}$ by the continuity of $X$ and $M$. Moreover $X_{r}^{(n)}$ are bounded by $\sup _{z \in T}\left\{\left(\left|M^{k}\right| \vee 1\right)|X|\right] \in \mathcal{L}^{1}\left(\mu^{r}, L_{0}(P)\right)$, then, by Theorem 2.4 we have that $\mu^{r}\left(1_{R_{x}} X M^{k-r}\right)=P-\lim _{n} \mu^{r}\left(X_{r}^{(n)}\right)$.

By continuity of $X$ and $M$ the hypotheses of Lemma 4.1 are satisfied and

$$
\mu^{r}\left(X_{r}^{(n)}\right)=\sum_{u \in S_{z}^{n}} X_{u} M_{u}^{k-r} \mu^{r}\left(I_{\Omega \times(u, \bar{u}]}\right)
$$

And so

$$
\begin{aligned}
& \mu^{(k)}\left(1_{R_{z}} X\right)=P-\lim _{n}\left[\sum_{r=1}^{k}\left(\begin{array}{c}
k \\
r
\end{array}\right)(-1)^{k-r}\left(\sum_{u \in S_{z}^{n}} X_{u} M_{u}^{k-r} \mu^{r}\left(1_{\Omega \times(u, \vec{u}}\right)\right]\right. \\
& =P-\lim _{n}\left(\sum_{u \in S_{z}^{n}} \sum_{r=1}^{k}\left(\begin{array}{c}
k \\
r
\end{array}\right)(-1)^{k-r} X_{u} M_{u}^{k-r} M^{r}\left(\Delta_{u}\right)\right) .
\end{aligned}
$$

By Lemma $3.3 \sum_{r=1}^{k}\left({ }_{r}^{k}\right)(-1)^{k-r} M_{u}^{k-r} M^{r}\left(\Delta_{u}\right)=\Delta_{u}^{k}(M)$, then the last limit is equal to $P-\lim _{n} \sum_{u \in S_{x}^{n}} X_{u} \Delta_{u}^{k}(M)$.

Corollary 4.3. If $M \in m_{c}^{4}$, for all $k \geq 5 \mu^{(k)} \equiv 0$.

Proof: It is an immediate consequence of the above proposition and Proposition 1.5 of [S]. 
Then, for $M \in m_{c}^{4}$, by the results of Section 2 and Theorem 4.7 of [A], the Itô's formula 2 is verified for $m=4$, i.e.

$$
(f \circ M)\left(\left(z, z^{\prime}\right]\right)=\sum_{r=1}^{4} \frac{1}{r !} \int_{\left(z, z^{\prime}\right)} f^{(r)}\left(M_{u}\right) d M_{u}^{(r)}
$$

and all the summands coincide with the terms of the Itô's formula 1.

Remark. As a consequence of the fact that $M^{(k)}$ and $\bar{M}^{(k)}$ define the same stochastic measure if $M \in m_{c}^{4}$, we have that the $k$-variations of a martingale that belongs to $m_{c}^{4}$ are $L_{0}(P)$-integrators in the sense of Bichteler. In [I2] Imkeller has studied the properties of $L_{p}(P)$-integrators that satisfy the $k$ variations of the martingales, and has proved a better result: if $M$ is a square integrable martingale then its 2 and 3 -variations are $L_{p}(P)$-integrators for some $p>0$.

\section{Appendix. Martingale inequalities}

In this appendix we state the versions of the martingale inequalities that have been used in this work. For example, in the Burkholder's inequalities, we do not consider the general case in which appears the norms in the Orlicz space $L^{\Phi}$, with $\Phi$ a Young's function (see [DM] for the definition of a Young's function, and for the general version of the inequalities in the one-parameter case).

We first state the one-parameter inequalities.

If $\left\{X_{t}, t \in T\right\}$ is a process, with $T$ an arbitrary parameter set, we define $X^{*}=\sup _{t \varepsilon T}\left|X_{t}\right|$.

Maximal inequality. Let $\left\{X_{t}, t \in \mathbb{R}_{+}\right\}$be a positive submartingale. Then, for all $p>1$ we have that

$$
\left\|X^{*}\right\|_{L^{p}(\Omega)} \leq q \sup _{1 \in \mathbb{R}_{+}}\left\|X_{t}\right\|_{L^{p}(\Omega)}, \quad \text { where } \quad \frac{1}{p}+\frac{1}{q}=1 .
$$

If $X$ is a local martingale we denote $[X, X]$ the unique process such that

(1) $X^{2}-[X, X]$ is a local martingale,

(2) $\Delta[X, X]=(\Delta X)^{2}$, where $(\Delta X)_{t}=X_{t}-X_{t^{-}}$.

In the case in which $X$ is a square integrable martingale $[X, X]$ is the quadratic variation of $X$.

Inequalities of Burholder-Davis. If $\left\{X_{t}, t \in \mathbb{R}_{+}\right\}$is a local martingale, then for all $1 \leq p<\infty$

$$
\frac{1}{4 p}\left\|X^{*}\right\|_{L^{p}(\Omega)} \leq\left\|[X, X]_{\infty}^{\frac{1}{2}}\right\|_{L^{p}(\Omega)} \leq 6 p\left\|X^{*}\right\|_{L^{p}(\Omega)}
$$


When $p \in(1, \infty)$ these inequalities are called Burkholder inequalities. The Davis inequality concerns the case $p=1$.

If $\left\{A_{t}, t \in \mathbf{R}_{+}\right\}$is a predictable increasing process, we define the potential associated with $A$ as the positive submartingale $Z_{t}=E\left[A_{\infty} / \mathcal{F}_{t}\right]-A_{t}$ (where we have taken a right continuous with left-limits version of the martingale $\left.E\left[A_{\infty} / \mathcal{F}_{t}\right]\right)$

Inequality of Garsia-Neveu. Let $\left\{A_{t}, t \in \mathbb{R}_{+}\right\}$be a predictable increasing process such that the potential associated with $A, Z$, is bounded by a right continuous, with left limits martingale $M_{t}=E\left[M_{\infty} / \mathcal{F}_{t}\right]$. Then for $p \geq 1$

$$
\left\|A_{\infty}\right\|_{L^{p}(\Omega)} \leq p\left\|M_{\infty}\right\|_{L^{p}(\Omega)} .
$$

We now consider the two-parameter martingale inequalities.

Maximal inequality. (Cairoli) (see [C]) Let $\left\{M_{z}, \mathcal{F}_{z}, z \in \mathbb{R}_{+}^{2}\right\}$ be a separable martingale. Then

$$
\left\|M^{*}\right\|_{L^{p}(\Omega)} \leq q^{2} \sup _{z \in \mathbb{R}_{+}^{2}}\left\|M_{z}\right\|_{L^{p}(\Omega)}
$$

For the Burkholder's inequalities, we first consider the case of discrete parameter set.

If $M=\left\{M_{n, m}, \mathcal{F}_{n, m},(n, m) \in \mathbf{N}^{2}\right\}$ is a discrete martingale which vanishes on the axes, we define

$$
\begin{aligned}
& d_{n, m}=M(n, m)-M(n-1, m)-M(n, m-1)+M(n-1, m-1) \\
& S_{n, m}(M)=\left(\sum_{i=1}^{n} \sum_{j=1}^{m} d_{i, j}^{2}\right)^{1 / 2} \\
& S(M)=\left(\sum_{i=1}^{\infty} \sum_{j=1}^{\infty} d_{i, j}^{2}\right)^{1 / 2} .
\end{aligned}
$$

Theorem. $\forall p>1$ there exists constants $C_{p}, C_{p}^{\prime}$ (only dependent on $p$ ) such that

(1) $C_{p} E|S(M)|^{p} \leq \sup _{n, m} E\left|M_{n, m}\right|^{p} \leq C_{p}^{\prime} E|S(M)|^{p}$, see $[\mathbf{M}]$.

For $p=1$, we have that

(2) $\sup _{m} E\left[\sup _{n}\left|M_{n, m}\right|\right] \leq C E[S(M)]$, see $[\mathrm{L}]$, and

(3) If $M$ is a strong martingale, there exist constans $C_{1}$ and $C_{1}^{\prime}$ such that $C_{1} E[S(M)] \leq E\left[\sup _{n, m}\left|M_{n, m}\right|\right] \leq C_{1}^{\prime} E[S(M)]$, see $[\mathbf{B r}]$ for the first inequality, and $[\mathbf{F I}]$ for the second one. 
It is possible, under conditions of continuity and $L^{p}$-majoration of the martingale, to pass to the limit the inequalities of the last theorem. If a martingale $M$ is bounded in $L^{p}, p \geq 2$, then it is closed and we can consider the terminal variable $M_{\infty, \infty}$ (if we have the martingale on $R_{z_{0}}, M_{\infty, \infty}$ coincides with $M_{z_{0}}$ ).

Theorem. (see.[N3]). Let $\left\{M_{z}, z \in \mathbb{R}_{+}^{2}\right\}$ be a martingale belonging to $m_{c}^{p}$, $p \geq 2$. Then there exist $C_{p}, C_{p}{ }^{\prime}>0$ such that

(1) $\left.C_{p} E \mid<M>_{\infty, \infty}^{p / 2}\right] \leq E\left[\sup _{z}\left|M_{z}\right|^{p}\right] \leq C_{p}^{\prime} E\left(<M>_{\infty, \infty}^{p / 2}\right)$,

(2) $C E\left[\left|M_{\infty, \infty}\right|\right] \leq E\left[<M>_{\infty, \infty}^{1 / 2}\right]$.

Acknowledgement. I wish to thank M. Sanz for having proposed this problem and for her useful suggestions.

\section{References}

[A] Allain, M.F., Semimartingales indexées par une partie de $\mathbb{B}^{d}$ et formule d'Itô. Cas continu, ZfW 65 (1984), 421-444.

[B] BICHTELER, K., Stochastic integration and $L_{p}$ theory of semimartingales, Ann. of Probability 9, 1 (1981), 49-89.

$[\mathrm{Br}] \mathrm{BROSSARD}, I$, Régularité des martingales à deux indices el inegalités des normes, in "Processus Aléatoires à Deux Indices (Colloque ENST-CNET, Paris 1980)." Lect. Notes in Math. 863, 91-121, Springer-Verlag, Berlin 1981.

[C] CAIRoli, R., Une inegalité pour martingales à indices multiples et ses applications, in "Sem. de Prob. IV." Lect. Notes in Math. 124, 1-27, Springer-Verlag, Berlin 1970.

[CW] CAIRoli, R., WaLSH, B., Stochastic integrals in the plane, Acta Math. 134 (1975), 111-183.

[Ch] Chevalier, L., Martingales continues à deux paramètres, Bull. Sc. Math. , 2eme. Série 106 (1982), 19-62.

[DM] Deinacherie, C., Meyer, P. A., "Probabilités et Potentiel (Tome II)," Ed. Hermann, Paris, 1980.

[FI] Frangos, N., IMKELLER, P., Some inequalities for strong martingales, Ann. de l'Institut H. Poincaré 24, 3 (1988), 395-402.

[I1] IMkELLER, P., A stochastic Calculus for continuous $N$-parameter strong martingales, Stoch. Processes and their Appl. 20 (1985), 1-40.

[I2] IMKELLER, P., A class of two-parameter stochastic integrators. Preprint.

[L] LEDOUX, Inegalités de Burkholder pour martingales indexées par $N \times N$, in "Processus Aléatoires à Deux Indices (Colloque ENST-CNET, Paris 1980)." Lect. Notes in Math. 863, 122-127, Springer-Verlag, Berlin 1981. 
[M] Metraux, C., Quelques inegalités pour martingales à paramètre bidimensionel, in "Sem. de Prob. XII." Lect. Notes in Math. 649, 170-179, Springer-Verlag, Berlin 1978.

[MP] Métivier, M. ,Pellaumail, J., Mesures stochatiques à valeurs dans les espaces $L_{0}$, Zf $W 104$ (1979), 101-114.

[N1] NUALART, D., On the quadratic variation of two parameter continuos martingales, Ann. of Probab. 12 (1984), 445-457.

[N2] NualaRT, D., Une formule d'Itô pour les martingales continues à deux indices et quelques applications, Ann. de l'Institut H. Poincaré 20, 3 (1984), 251-275.

[N3] NUALART, D., Variations quadratiques et inćgalités pour les martingales à deux indices, Stochastics 15 (1985), 51-64.

[S] SANZ, M., $r$-Variations for two-parameter continuous martingales and Itô's formula, Stoch. Processes and their Appl. (to appear).

[WZ] WONG, E. ,ZAKAI, M., Differentiation formulas for stochastic integrals in the plane, Stoch. Processes and their Appl. 6 (1978), 339-349.

Departament de Matemàtiques
Universitat Autònoma de Barcelona
08193 Bellaterra (Barcelona)
SPAIN

Rebut el $\gamma$ de Març de 1989 